Estudios Geográficos, LXIX, 265, pp. 609-635, julio-diciembre 2008

ISSN: 0014-1496

elSSN: $1988-8546$

doi: 10.3989/estgeogr.0416

\title{
Beneficios y costes ambientales en la Directiva Marco del Agua: conceptos y estimación ${ }^{1}$
}

\section{Enviromental benefits and costs in the Water Framework Directive: concepts and estimation}

\author{
Julia Martín-Ortega ${ }^{\star}$ \\ $\mathrm{y}$ \\ J. Berbel ${ }^{\star \star}$
}

\section{INTRODUCCIÓN}

En la Declaración de Dublín de la Conferencia Internacional sobre Agua y Medio Ambiente celebrada en 1992, se reconoce formalmente el carácter económico del agua y la necesidad de que éste sea tenido en cuenta en su gestión. Sin embargo, en la política hídrica tradicional no se ha incluido el valor total de los recursos naturales y los costes relacionados con el daño ambiental de las medidas de gestión. Se ha reconocido tanto desde el ámbito científico (por ejemplo Pearce y Turner, 1995; Costanza et al., 1997; Birol et al., 2006a) como desde el ámbito institucional (UNESCO, 2002; Naciones Unidas, 2003; Unión Europea, 2000) que los recursos hídricos están siendo

1. Este artículo se enmarca dentro de las actividades de investigación del proyecto del VI Programa Marco de la UE: AquaMoney: Development and Testing of Practical Guidelines for the Assessment of Environmental and Resources Costs and Benefits in the WFD (SSPI-022723) y la tesis doctoral Beneficios ambientales no de mercado asociados a la implementación de la DMA: una aproximación territorial a la Demarcación Hidrográfica del Guadalquivir (MartínOrtega, 2008), financiada por el Plan de Andaluz de Investigación de la Junta de Andalucía mediante el programa de formación de doctores (convocatoria de junio de 2004).

* Investigadora del Departamento de Economía, Sociología y Política Agrarias de la Universidad de Córdoba. Email, es2maorj@uco.es

** Profesor titular del Departamento de Economía, Sociología y Política Agrarias de la Universidad de Córdoba. 
sobreexplotados y son objeto de una asignación ineficiente, debido, en parte, a que los costes asociados al daño ambiental y a los usuarios no son íntegramente contabilizados, ni es reconocido el valor económico total del agua en los procesos de toma de decisión.

Desde el ámbito institucional se han puesto en marcha iniciativas que tratan de fomentar una gestión sostenible y asegurar su conservación. En el caso de la Unión Europea, la protección del medio ambiente en general ha sido un tema clave de la legislación y ya desde el Tratado de Maastricht ${ }^{2}$ se hace mención específica a la protección ambiental orientada a la salvaguarda de la salud humana y el logro del desarrollo sostenible. En esta línea, a mediados de los años ochenta empezó a generarse un cierto consenso sobre la necesidad de establecer estrategias más completas e integradas en relación la regulación de los recursos naturales. En los años noventa comenzaron a surgir directivas horizontales que trataban de asegurar una protección más efectiva. Un ejemplo ilustrativo lo encontramos en la Directiva de Control y Prevención Integrado de la Contaminación (IPPC)3, que se diseña como un instrumento de prevención y minimización de emisiones a la tierra, al aire y al agua ${ }^{4}$.

En este contexto, la Unión Europea promulgó en el año 2000 la Directiva Marco de Agua $^{5}$ como resultado de una larga serie de iniciativas comunitarias que desde finales de los años ochenta habían ido poniendo de manifiesto la necesidad de una regulación integrada de las aguas (en el cuadro 1 puede verse un resumen de dichas iniciativas).

En su artículo primero la DMA establece como objetivo la protección de las aguas superficiales continentales, de transición y costeras y aguas subterráneas, lo cual implica la prevención del deterioro adicional de las aguas, la protección de los ecosistemas (acuáticos, humedales y terrestres dependientes de los ecosistemas acuáticos), la promoción del uso sostenible del agua, la disminución de vertidos de sustancias peligrosas y la prevención de la contaminación de aguas subterráneas, así como de los efectos de inundación y sequía. De este modo, la Directiva, además de simplificar la legislación previa, perfila una estrategia general para la gestión del agua en Europa con el objetivo de conseguir el buen estado ecológico para todas las aguas comunitarias para el año 2015.

2. DOC 191, 29 de junio de 1992.

3. Directiva 96/61/CE.

4. La Directiva IPPC, no obstante, se aplica sólo a determinadas actividades industriales (y actividades ganaderas intensivas) que se consideran susceptibles de presentar riesgos medioambientales concretos.

5. Directiva 2000/60/CE por la que se establece el marco de actuación en política de aguas. Boletín Oficial de las Comunidades Europeas, 22 de diciembre de 2000. 


\begin{tabular}{|c|c|c|c|}
\hline Año & Acontecimiento/organismo & Contenido & Referencia \\
\hline 1988 & $\begin{array}{l}\text { Seminario ministerial sobre política } \\
\text { de aguas (Francfort). }\end{array}$ & $\begin{array}{l}\text { Necesidad de una legislación } \\
\text { comunitaria que abordara } \\
\text { la calidad ecológica de las aguas. }\end{array}$ & $\begin{array}{l}\text { Resolución del Consejo } \\
\text { Europeo de } 28 \text { de junio } \\
\text { (DOC } 209 \text { de } 9 \text { de agosto } \\
\text { de 1988, página 3). }\end{array}$ \\
\hline 1990 & Reunión de ministros de la Haya. & $\begin{array}{l}\text { Urgencia por adoptar medidas } \\
\text { para evitar el deterioro de las } \\
\text { aguas dulces y se solicita } \\
\text { la aplicación de un programa } \\
\text { de medidas antes del año } 2000 .\end{array}$ & \\
\hline $\begin{array}{l}1992 \\
1995\end{array}$ & Consejo Europeo. & $\begin{array}{l}\text { Exigencias de actuación en materia } \\
\text { de aguas subterráneas. Revisión de } \\
\text { la Directiva } 80 / 68 / C E E \text { de } 17 \\
\text { de diciembre de } 1979 \text {. }\end{array}$ & $\begin{array}{l}\text { DOC } 59 \text { de } 6 \text { de marzo de } \\
\text { 1992, página } 2 \text { y DOC } 49 \\
\text { de } 28 \text { de febrero de } 1995 .\end{array}$ \\
\hline 1995 & Agencia Europea de Medio Ambiente. & $\begin{array}{l}\text { Necesidad de tomar medidas para } \\
\text { proteger las aguas comunitarias } \\
\text { tanto en términos cualitativos } \\
\text { como cuantitativos. }\end{array}$ & $\begin{array}{l}\text { Informe "El medio ambiente } \\
\text { en la Unión Europea" } \\
\text { (Agencia Europea de Medio } \\
\text { Ambiente, 1995). }\end{array}$ \\
\hline 1996 & $\begin{array}{l}\text { Consejo Europeo, Comité de las } \\
\text { Regiones, Comité Económico y Social } \\
\text { y Parlamento Europeo. }\end{array}$ & $\begin{array}{l}\text { Solicitudes explícitas de } \\
\text { elaboración de una directiva } \\
\text { marco de aguas. }\end{array}$ & \\
\hline
\end{tabular}

Fuente: Elaboración propia.

Según Gómez (2006), en la DMA subyace el principio según el cual gestionar el agua no se reduce a arbitrar los mecanismos que sean necesarios para atender a corto plazo las demandas de servicios del agua que puedan surgir de los distintos sectores de la economía, sino que se entiende que los ecosistemas hídricos constituyen un patrimonio colectivo que debe preservarse haciendo que todos los usos que supongan una presión sobre el medio ambiente sean compatibles con la mejora de los ecosistemas hídricos.

Para la consecución de sus objetivos ecológicos, la DMA prescribe de forma bastante novedosa la utilización de principios y herramientas económicas, respondiendo a la necesidad de una regulación integrada de las aguas para alcanzar una gestión sostenible de los recursos hídricos y hacer frente a la creciente presión en la demanda de agua de buena calidad. Esta importancia de los aspectos económicos no debe, sin embargo, ser interpretada como una primacía de un enfoque economicista para la gestión hídrica. Todo lo contrario, en el contexto de la DMA la economía es una herramienta subordinada a la consecución del objetivo ecológico (Ferrer y La Roca, 2006; Gómez, 2006). En términos generales, el papel del análisis económico en el proceso de implementación de la DMA es el de proveedor 
de información y soporte del proceso participativo de toma de decisiones (Comisión Europea, 2003a).

Dentro de los aspectos económicos de la DMA, tienen particular relevancia las nociones de beneficio y coste ambiental. Esto supone la primera inclusión de estos conceptos en la legislación ambiental europea. Sin embargo, su definición no ha sido todavía suficientemente clarificada ni desde el ámbito institucional ni desde el ámbito científico, lo cual dificulta obviamente su estimación. De hecho, hasta el momento los Estados Miembros no han realizado adelantos significativos en este sentido.

Teniendo en cuenta la falta de una definición clara y explícita de los beneficios ambientales en el texto de la Directiva y las distintas interpretaciones que se han realizado, unidas a la necesidad acuciante de evaluar la desproporcionalidad de los costes de la implementación de la DMA (los Planes de cuenca elaborados de acuerdo la nueva legislación deberán estar publicados a finales de 2009), consideramos necesario establecer desde el ámbito científico-académico una definición práctica de los beneficios y costes ambientales en el contexto de la DMA e identificar los elementos clave para su estimación, de modo que estos valores puedan ser incorporados como insumos de los procesos de decisión de la política hídrica.

El objetivo de este artículo es el de contribuir a la reflexión sobre los conceptos de beneficio y coste ambiental contexto de la Directiva, discutiendo las interpretaciones que de estos conceptos se han hecho hasta el momento y planteando una definición práctica. Repasamos los avances que se han realizado en el ámbito Europeo en su evaluación y tratamos de clarificar las bases para su estimación en el proceso de implementación de la DMA.

\section{Beneficios y Costes AMBIENTALES EN LA DMA}

A pesar de que no se ha prestado hasta el momento especial atención por parte de los Estados Miembros a la estimación de los beneficios ambientales en el proceso de implementación de la DMA (World Wide Fund for Nature and European Bureau 2006), su papel es crucial para la meta última de la norma Europea. El artículo 4 de la DMA, en el que se fijan los objetivos ambientales de la Directiva, permite la derogación de dichos objetivos cuando éstos no pueden conseguirse por motivos de viabilidad técnica o costes desproporcionados. Las excepciones posibles en los objetivos de la DMA pueden considerarse de dos tipos distintos: que los costes 
sean desproporcionados respecto a la capacidad financiera para asumirlos, o que los costes del plan sean desproporcionados respecto a los beneficios que supone la mejora en la calidad ambiental. En ambos casos, corresponde a los Estados Miembros determinar y probar la desproporcionalidad de los costes.

En el primer caso, se trata es de un problema de capacidad financiera que depende del modo en que se repercutan los costes del Plan de cuenca sobre los usos del agua, y que justificaría el aplazamiento de los objetivos ambientales (Gómez, 2006). En el segundo caso, la desproporción es relativa a la bondad de las ventajas de los planes de cuenca en relación con sus costes y puede conllevar a una posible rebaja de los objetivos del buen estado. Mientras que en el caso anterior los mecanismos financieros pueden ser suficientes para la derogación temporal de los mismos, en el caso de la rebaja de los objetivos será necesario realizar un análisis coste-beneficio en el que se incluyan los costes y beneficios sociales completos asociados con las medidas. A la postre, se tratará de una decisión política en la que no siempre será obvio identificar la desproporcionalidad, pero en cualquier caso, será premisa necesaria haber estimado los beneficios totales derivados del buen estado ecológico, incluidos los beneficios ambientales que no tienen reflejo en el mercado.

Los costes ambientales son solicitados además por la DMA de manera explícita en el artículo 9, cuando se prescribe la recuperación completa de los costes de los servicios del agua, entre los que se incluyen manifiestamente los costes ambientales y del recurso ${ }^{6}$. Esta estimación del grado de recuperación de costes tiene como objetivo servir de base a políticas de precios que proporcionen un estímulo necesario para un uso más eficiente del recurso por parte de los usuarios. Esto supone la búsqueda de una adecuada contribución de todos los usuarios del agua a la recuperación de los costes del servicio de la misma, es decir, supone la aplicación práctica del principio de "quien contamina paga" o "quien usa el recurso paga" y requiere necesariamente la incorporación de los costes ambientales y del recurso.

La introducción de los beneficios y costes ambientales en la DMA supone la primera inclusión de estos conceptos en la legislación ambiental europea. Sin embargo, su definición no ha sido todavía suficientemente

6. Artículo 9 de la DMA, epígrafe 1: Los Estados miembros tendrán en cuenta el principio de recuperación de costes de los servicios del agua, incluyendo los costes ambientales y del recurso, considerando el análisis económico llevado a cabo de acuerdo con el Anexo III, y de particularmente de acuerdo con el principio de "quien contamina paga". 
clarificada ni desde el ámbito institucional ni desde el ámbito científico. Según recuerdan Ferrer y la Roca (2006a), ya en el año 2000 Aguilera Klimt advertía de los problemas derivados de la imprecisa noción de recuperación de costes en el texto de la Directiva.

La guía WATECO (Comisión Europea, 2003a)7 define los costes ambientales como los costes derivados del daño que los usos del agua imponen sobre el ambiente y los ecosistemas y sus usuarios, por ejemplo el coste asociado a la disminución de la calidad ecológica por contaminación de un ecosistema acuático, o la degradación de suelo por salinización. En las reflexiones posteriores que se llevaron a cabo en el seno de la Estrategia Conjunta de Implementación de la DMA (hojas informativas del grupo de trabajo ECO2, encargado de desarrollar la guía WATECO), los costes ambientales fueron definidos como el valor económico del daño ambiental originado por la degradación y agotamiento (depleción) del recurso, como consecuencia de su uso (por ejemplo extracción de agua o emisión de contaminantes).

En ambos casos entendemos que la sociedad obtiene beneficios del buen estado ecológico de las aguas (beneficios ambientales) y que los costes ambientales se corresponden con los "beneficios perdidos" (coste de oportunidad) de no alcanzar dicho estado.

Como hemos mencionado, la Directiva hace referencia, además de a la noción de beneficio y coste ambiental, a la "del recurso", concepto que ha dado lugar a diferentes interpretaciones. Por su parte, WATECO define el coste del recurso como el coste de oportunidad de utilizar el recurso escaso agua para un determinado uso más allá de la capacidad natural de recarga o recuperación (por ejemplo sobreexplotación de un acuífero). Sin embargo, la definición de los costes del recurso proporcionada posteriormente por ECO2 puso el énfasis en la diferencia entre el valor económico del actual uso del agua y el valor económico de la mejor alternativa de uso posible. De este modo, según ECO2, los costes del recurso surgen si la alternativa de uso del agua genera mayor valor económico que el uso actual o previsto, pero no se restringe al caso de degradación o depleción del recurso. Es decir, para ECO2, los costes del recurso derivan de una situación de asignación ineficiente de los recursos, por encima de la idea de sobreexplotación del recurso que señalaba WATECO.

7. Guía no legalmente vinculante de carácter práctico elaborada para ayudar a la implementación de los aspectos económicos de la Directiva. 
Además de estas diferentes interpretaciones de coste del recurso, la propia distinción de los beneficios y costes en "ambientales" y "del recurso" no es tan evidente. Por su parte, Schaafsma y Brouwer (2006) no encuentran, en su exhaustiva revisión de la literatura científica a este respecto, referencias a esta distinción conceptual. Para Ferrer y la Roca (2006a) la interpretación de los costes ambientales y del recurso como categorías separables está en el origen de la confusión conceptual en el contexto de la Directiva. Por nuestra parte, diremos que la distinción que entre coste y beneficio ambiental y del recurso hacen tanto WATECO como ECO2 parece estar basada en la noción de coste de oportunidad, asociado a la existencia de una asignación alternativa en el caso de los costes del recurso. Sin embargo, convendría ser prudente a la hora de asumir esta distinción ya que en la noción de coste total va incluida la noción de coste de oportunidad de modo que el coste ambiental (derivado de un daño ambiental) también puede llevar asociado un determinado coste de oportunidad (por ejemplo, si el agua tiene mala calidad como consecuencia de la contaminación no podrá utilizarse para un determinado uso, generándose un coste de oportunidad).

Una interpretación alternativa de la distinción entre ambos conceptos, y por la que apostamos en este artículo, podría situarse en las condiciones de la asignación. En esta línea, los costes del recurso surgirían como consecuencia de la competencia entre los usos humanos en relación con el medio ambiente como resultado de su limitada disponibilidad en términos cuantitativos. El beneficio del recurso sería, pues, el bienestar derivado del mantenimiento de niveles de agua favorable al buen funcionamiento del ecosistema en situaciones de escasez (valor de "asignar agua la medio ambiente" frente a usos alternativos). Mientras que los beneficios ambientales se referirían al bienestar asociado al estado ecológico de la masa de agua o de la demarcación como resultado de su nivel de calidad. De este modo, los beneficios y costes del recurso recogerían la dimensión cuantitativa del asunto en las situaciones en las que la cantidad es un problema económico (situaciones de escasez y por tanto problema de asignación) y los beneficios y costes ambientales, la dimensión cualitativa. En esta distinción debe sin embargo tenerse en cuenta que ambas dimensiones (cuantitativa y cualitativa) no son estrictamente independientes, ya que obviamente existe una estrecha relación entre cantidad (disponibilidad) de agua y su calidad (es decir, para mantener ciertos niveles de calidad es necesario un cierto caudal).

Abogamos pues aquí por la definición práctica del beneficio ambiental como el valor de mercado y no de mercado (ganancia de bienestar) de 
los bienes y servicios proporcionados por la Demarcación Hidrográfica como consecuencia de la consecución del buen estado ecológico, es decir, por el valor económico total concedido por la sociedad a tal estado, incluidos los valores de uso y de no uso. El coste ambiental, siguiendo la filosofía de la interpretación económica de la DMA reflejada en WATECO, quedaría definido como el coste económico total (pérdida de bienestar) derivado del daño ambiental a un ecosistema acuático como resultado del estado físico y/o ecológico del sistema hídrico, es decir, el coste de oportunidad por la no consecución del buen estado ecológico. La clarificación en términos prácticos de esta noción determinará de forma esencial la estimación que de los beneficios ambientales se haga en aplicación de la DMA, asunto sobre el que discutimos en mayor profundidad más adelante, tras un repaso a los avances que se han realizado en Europa hasta el momento.

\section{AVANCE EUROPEO EN LA ESTIMACIÓN DE LOS BENEFICIOS Y COSTES AMBIENTALES DE LA DMA}

Se asume de forma generalizada que la implementación de la DMA va a originar beneficios sustanciales pero, como constatan Bateman et al. (2006a), se han realizado hasta el momento escasas valoraciones económicas de estos beneficios, en particular en lo que concierne a los valores que no tienen mercado.

Quizá en parte a causa de la falta de una definición clara de estos conceptos y en general, como consecuencia de la gran cantidad de información que ha sido necesario movilizar en las primeras fases de implementación de la DMA, lo cierto es que hasta el momento los Estados miembros no han realizado avances significativos en la estimación de los beneficios y costes ambientales. La mencionada encuesta realizada por el World Wide Fund for Nature and European Bureau (2006) pone de manifiesto la poca atención que se ha prestado en la primera fase de implementación de la Directiva a estos aspectos, limitándose en la mayor parte de los casos a una mera descripción de los impuestos ambientales existentes.

Strosser (2006) recoge ejemplos de estudios y actividades relativas a la evaluación de los costes ambientales y del recurso llevados a cabo en el contexto de aplicación de la DMA en distintos países de la Unión Europea (cuadro 2). 


\section{CUADRO 2. ESTUDIOS EUROPEOS RELACIONADOS CON LA ESTIMACIÓN DE BENEFICIOS Y COSTES AMBIENTALES EN EL CONTEXTO DE LA DMA}

País Aspectos evaluados Metodología $\quad$ Contexto/observaciones

\begin{tabular}{|c|c|c|c|}
\hline $\begin{array}{l}\text { Países } \\
\text { Bálticos }\end{array}$ & $\begin{array}{l}\text { Continuidad del río, migración } \\
\text { de peces y eutrofización. }\end{array}$ & VC. & Proyecto ENCOBALT (2007). \\
\hline Francia & Varios. & VC y otros. & $\begin{array}{l}\text { Revisión de estudios } \\
\text { existentes y desarrollo } \\
\text { de guías para TB. }\end{array}$ \\
\hline \multirow[t]{2}{*}{ Alemania } & $\begin{array}{l}\text { Hidromorfología, difusión de la } \\
\text { contaminación en aguas superficiales, } \\
\text { retención de nutrientes, } \\
\text { biodiversidad. }\end{array}$ & Costes reposición, TB. & $\begin{array}{l}\text { Demarcaciones del Weser } \\
\text { y Wera (escala Länder). } \\
\text { Desarrollo de un manual } \\
\text { práctico. }\end{array}$ \\
\hline & Tasas e impuestos ambientales. & Recopilación de datos existentes. & $\begin{array}{l}\text { Informe de recuperación } \\
\text { de costes. Estudios de caso: } \\
\text { subcuenca de Mittelrhein, } \\
\text { subcuenca de Lippe y región } \\
\text { de Leipzig. }\end{array}$ \\
\hline 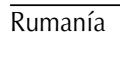 & Contaminación superficial. & No especificado. & $\begin{array}{l}\text { Estudio piloto para la } \\
\text { Demarcación del Bistrita. }\end{array}$ \\
\hline $\begin{array}{l}\text { Bélgica, } \\
\text { Holanda } \\
\text { y Francia }\end{array}$ & Aguas superficiales y costeras. & VC. & $\begin{array}{l}\text { Desarrollado en el contexto } \\
\text { de la Comisión Internacional } \\
\text { del Scheldt. }\end{array}$ \\
\hline \multirow[t]{2}{*}{ Holanda } & $\begin{array}{l}\text { Restauración ecológica, mejora } \\
\text { de agua de baño, mejora de la } \\
\text { calidad del agua. }\end{array}$ & Preferencias expresadas. & $\begin{array}{l}\text { Brouwer (2006b), Brouwer } \\
\text { (2008). }\end{array}$ \\
\hline & $\begin{array}{l}\text { Costes de las medidas de } \\
\text { recuperación de la calidad. }\end{array}$ & Estimación costes. & $\begin{array}{l}\text { Informe de recuperación } \\
\text { de costes (artículo } 9 \text { DMA). }\end{array}$ \\
\hline Eslovenia & $\begin{array}{l}\text { Contaminación por nitratos } \\
\text { y pesticidas en aguas subterráneas. }\end{array}$ & VC. & $\begin{array}{l}\text { Estudio piloto para la } \\
\text { Demarcación del Krka. } \\
\text { Financiado por la UE } \\
\text { (BRIDGE). }\end{array}$ \\
\hline \multirow[t]{3}{*}{$\begin{array}{l}\text { España } \\
\text { nan }\end{array}$} & Aguas superficiales. & $\begin{array}{l}\text { EE y mecanismos de participación } \\
\text { pública. }\end{array}$ & $\begin{array}{l}\text { Plan de cuenca del río Cidacos. } \\
\text { Aplicación de la Guía de } \\
\text { Análisis Económico de la DMA, } \\
\text { Álvarez-Farizo y Hanley } \\
\text { (2006) y Álvarez-Farizo et al. } \\
\text { (2006). }\end{array}$ \\
\hline & $\begin{array}{l}\text { Coste de oportunidad de } \\
\text { mantenimiento de caudales } \\
\text { mínimos. }\end{array}$ & Modelización hidroeconómica. & $\begin{array}{l}\text { Desarrollo de herramientas } \\
\text { para el análisis económico de } \\
\text { sistemas de recursos hídricos } \\
\text { en aplicación de la DMA } \\
\text { (Andreu et al., 2006). }\end{array}$ \\
\hline & $\begin{array}{l}\text { Beneficios ambientales de la mejora } \\
\text { de la calidad del agua. }\end{array}$ & VC y EE. & $\begin{array}{l}\text { Aplicación a la Demarcación } \\
\text { Hidrográfica del Guadalquivir } \\
\text { (Martín-Ortega et al. en prensa) }\end{array}$ \\
\hline Suecia & $\begin{array}{l}\text { Continuidad, ecología, } \\
\text { hidromorfología y energía } \\
\text { hidráulica. }\end{array}$ & EE. & $\begin{array}{l}\text { Iniciativa de la Agencia } \\
\text { Sueca de Protección del } \\
\text { Medio Ambiente en el } \\
\text { contexto de la identificación } \\
\text { de masas de agua altamente } \\
\text { modificadas. }\end{array}$ \\
\hline
\end{tabular}


CuAdro 2. Estudios EuROPEOS RELACIONADOS CON LA ESTIMACIÓN DE BENEFICIOS Y COSTES AMBIENTALES EN EL CONTEXTO DE LA DMA (CONT.)

\begin{tabular}{|c|c|c|c|}
\hline País & Aspectos evaluados & Metodología & Contexto/observaciones \\
\hline \multirow[t]{3}{*}{$\begin{array}{l}\text { Reino } \\
\text { Unido }\end{array}$} & $\begin{array}{l}\text { Costes desproporcionados de } \\
\text { la DMA. }\end{array}$ & Preferencia expresada y TB. & $\begin{array}{l}\text { DEFRA. Análisis } \\
\text { coste-eficacia y desarrollo } \\
\text { de metodologías para la } \\
\text { evaluación de costes } \\
\text { desproporcionados. }\end{array}$ \\
\hline & $\begin{array}{l}\text { Valor de la mejora de la calidad } \\
\text { ecológica. }\end{array}$ & $\mathrm{EE}$. & $\begin{array}{l}\text { Cuencas Motray y Brothock } \\
\text { (Escocia) (Hanley et al., } \\
\text { 2006). }\end{array}$ \\
\hline & $\begin{array}{l}\text { Beneficios de los programas } \\
\text { de medida de la DMA en Inglaterra } \\
\text { y Gales. }\end{array}$ & EE. & $\begin{array}{l}\text { Proyecto de investigación } \\
\text { (DEFRA re CRP Project 4b/c) } \\
\text { (Baket et al., 2007). }\end{array}$ \\
\hline
\end{tabular}

Siglas: VC: valoración contingente, EE: experimentos de elección y TB: transferencia de beneficios. Fuente: Ampliado de Strosser (2006).

El proyecto ENCOBALT (2007), iniciado en agosto de 2006, estaba destinado no tanto a la estimación de los beneficios y costes ambientales como a la capacitación de los Países Bálticos para su evaluación en el futuro próximo. El proyecto tenía además el objetivo de promover el interés de los agentes decisores y grupos involucrados en la gestión de las demarcaciones hidrográficas, así como crear una red de expertos entre los tres Países Bálticos.

En el caso de Alemania, en el año 2006 fue elaborado un informe (Görlach e Interwies, 2006) en el que se reconoce el escaso trabajo que se había realizado hasta el momento desde el ámbito institucional en relación a este asunto. Los autores llegan incluso a afirmar que en algunos de los estados federales alemanes existe la percepción de que los beneficios y costes ambientales no son un asunto fundamental y que no jugarán un papel decisivo en la recuperación de los costes de los servicios del agua o en el análisis coste eficacia de las medidas. El manual de la Agencia Federal de Medio Ambiente (Interwies et al., 2004) considera que los costes ambientales y del recurso del suministro del agua en Alemania son bajos y que sólo pueden ser significativos (los del recurso) en aquellas zonas con escasez de agua. De hecho, en los tres estudios pilotos elegidos para el análisis de la recuperación de costes (subcuenca de Mittelrhein, sub-cuenca de Lippe y la región de Leipzig), sólo se han considerado los costes ambientales y del recurso previamente internalizados, es decir, los costes que están cubiertos por las tasas e impuestos ambientales (costes de tratamiento de aguas residuales y algunos pagos para la compensación de la preservación de la naturaleza y la pesca recreativa). 
Brouwer (2006b) repasa los estudios de valoración económica mediante preferencias expresadas de los cambios de la calidad del agua que se han Ilevado a cabo en los Países Bajos, fundamentalmente como parte del proceso de revisión de la Directiva de Aguas de Baño y la implementación de la Directiva Marco del Agua. Estos estudios incluyen la valoración de la restauración ecológica del lago Frisian, valoración de la mejora de la calidad del agua de baño y valoración de la limpieza de sedimentos contaminados y preservación de la biodiversidad. Uno de los trabajos mencionados por Brouwer y realizado por él mismo estaba destinado a determinar la disponibilidad a pagar de los holandeses (5.000 entrevistas fueron realizadas aleatoriamente por todo el país) a mejorar de la calidad del agua como una primera estimación de la desproporcionalidad de costes en el contexto de la DMA (Brouwer, 2008).

También en los Países Bajos, pero en colaboración con Francia y Bélgica en el contexto de la Comisión Internacional del río Scheldt, se ha evaluado la mejora de la calidad de las aguas superficiales y costeras mediante valoración por preferencias expresadas (Schaafsma et al., en prensa).

En 2004 el Departamento de Medio Ambiente, Alimentación y Asuntos Rurales (DEFRA) de Reino Unido encargó un informe sobre las metodologías para el análisis coste-eficacia y para la evaluación de los costes desproporcionados (Postle et al., 2004). Por su parte, Hanley et al. (2006a) Ilevaron a cabo una investigación para la estimación de los beneficios ambientales derivados de la mejora del estado del agua en Escocia. Además, recientemente se ha hecho público el informe sobre los beneficios derivados de la DMA encargado también por la DEFRA en el que se aplican métodos de preferencias expresadas (Baker et al., 2007). Se entrevistaron 1.500 hogares en 50 localidades aleatoriamente distribuidas y estratificadas por tamaño de población y cuenca en Inglaterra y Gales en Julio de 2007. En el momento de redacción de este artículo, está en marcha el proyecto $\mathrm{ChREAM}^{8}$ que analiza los costes y beneficios en agricultura derivados de la implementación de la DMA en la cuenca del río Humber en Inglaterra (Bateman et al., 2006a). El proyecto integra elementos hidrológicos, económicos, agronómicos y geográficos para analizar los efectos de la mejora de la calidad de agua impuesta por la Directiva sobre los ingresos de los agricultores.

El entonces Ministerio de Medio Ambiente de España se comprometió en el año 2000 en el marco de la Estrategia Común de Aplicación de la

8. The Catchment's Hydrology, Resources, Economics and Management. 
Directiva Marco del Agua a realizar un estudio piloto en el río Cidacos con el objetivo de ilustrar los diferentes elementos del análisis económico propuesto para la aplicación de la DMA, especialmente en el desarrollo del análisis coste-eficacia de las medidas para alcanzar los objetivos (artículo 11). Como resultado de ese compromiso se publicó en el año 2002 un informe sobre el análisis económico del Plan de Cuenca del río Cidacos (Ministerio de Medio Ambiente y Gobierno de Navarra, 2002). En dicho informe se hace, en primer lugar, un repaso cualitativo de los beneficios ambientales potenciales asociados a las medidas de mejora de la calidad de las aguas: beneficios relacionados con las actividades recreativas, con las actividades productivas y finalmente relacionados con la conservación o restauración de los servicios asociados con al ecosistema fluvial. Entre ellos se concede particular importancia al desarrollo de la actividad turística y parece tomarse en menor consideración los valores de no-uso. Por su parte, los trabajos de Álvarez-Farizo y Hanley (2006) y Álvarez-Farizo et al. (2006) tienen su origen en el proceso de consulta y participación pública desarrollados durante la elaboración del mencionado Plan de Cuenca del Río Cidacos.

Conviene señalar además señalar en España, los trabajos de modelización hidro-económica propuestos por Andreu et al. (2006), quienes integran recursos, infraestructuras y demandas a escala de cuenca o sistema de explotación mediante mecanismos de simulación y optimización para la estimación de los costes marginales de oportunidad asociados al mantenimiento de caudales mínimos y restricciones ambientales. En estos modelos, los autores incorporan funciones de valor económico del agua en los distintos usos, además de los costes variables de operación del sistema. Sin embargo, parece que las funciones de demanda que utilizan estos autores y que expresan la relación entre la demanda de agua suministrada y su valor marginal, incluyen exclusivamente usos productivos del agua.

A todos los trabajos anteriormente citados hay que añadir los que están actualmente representados en el proyecto de investigación AquaMoney 9 (Reino Unido, Noruega, España, Francia, Grecia, Hungría, Lituania, Bélgica, Italia, Dinamarca, Rumanía y Austria) que tiene como objetivo el desarrollo de guías prácticas para la valoración económica de los beneficios y costes ambientales en el contexto de la DMA.

9. Proyecto de investigación del $6^{\circ}$ Programa Marco de la UE: AquaMoney (SSPI022723): Guidelines for the assessment of environmental and resource costs and benefits of the Water Framework Directive (www.aquamoney.org). 
En cualquier caso, la mayoría de los trabajos aquí señalados (salvo el caso británico) proceden del ámbito académico o tienen un carácter experimental. Hay que señalar que en España acaba de publicarse recientemente la Instrucción de Planificación Hidrológica ${ }^{10}$ en la que se incluye la definición oficial de estos conceptos y que discutimos a continuación.

\section{ESTIMACIÓN DE BENEFICIOS Y COSTES AMBIENTALES: ENFOQUE DE DEMANDA}

De la definición práctica de los beneficios ambientales como los valores de los bienes y servicios proporcionados por la demarcación hidrográfica como consecuencia del buen estado ecológico de las aguas, se desprende que su estimación debe basarse en la estimación de la mejora del bienestar por pasar de la situación actual de la demarcación hidrográfica a una situación de buen estado ecológico. Esta aproximación responde a la concepción neoclásica en la que valor económico surge de la interacción entre un sujeto y un objeto (bien ambiental) como expresión de las preferencias individuales, asumiendo que estas preferencias son el reflejo de la maximización de la utilidad que mueve al individuo y que los cambios en la provisión del bien impactan en el bienestar individual.

Este enfoque encontrará detractores entre los seguidores de una visión más ecocéntrica de la preocupación ambiental. La llamada economía ecológica considera que el sistema económico forma parte de otro sistema mayor, la biosfera y utiliza mecanismos e instrumentos de contabilidad generalmente expresados en términos físicos, como los balances de masa y energía, para reflejar elementos económicos ${ }^{11}$.

Sin embargo, no debe entenderse que el enfoque aquí propuesto obvia la dimensión ecológica-ambiental: ésta forma parte esencial de la propuesta, ya que el objetivo último, en coherencia con la DMA, es la consecución del buen estado ecológico de las masas de agua. De este modo las necesidades sociales y ecológicas van indisolublemente aparejadas, contrariamente a como se ha tratado tradicionalmente la gestión ambiental (Wittmer et al., 2006). Esta propuesta no rechaza necesariamente la consideración de los valores intrínsecos (no instrumentales) reclamados por la economía ecológica, así como la llamada a la

10. Orden ARM/2656/2008 de 10 septiembre por la que se aprueba la instrucción de la planificación hidrológica (BOE núm. 229, de 22 de septiembre de 2008).

11. Para una mayor profundización sobre la economía ecológica recomendamos la lectura de Costanza (1989) y Martínez Alier y Roca Jusmet (2000). 
reflexión colectiva para la valoración de la herencia común de Azqueta y Delacámara (2006), simplemente busca soluciones adaptadas a los requerimientos y cronología de implementación de la Directiva.

En este sentido, es muy importante que las valoraciones económicas que se hagan de los beneficios y costes ambientales incluyan el valor económico total del agua y no sólo los valores de uso directo que tienen reflejo en el mercado. En el cuadro 3 se presenta un resumen de la "taxonomía" de los valores de los recursos hídricos.

\section{Cuadro 3. Componentes del VET de lOS RECURSOS Hídricos}

\begin{tabular}{lll} 
Valores de uso directo & \multicolumn{1}{c}{ Valores de uso indirecto } & \multicolumn{1}{c}{ Valores de opción } \\
\hline Agricultura de regadío. & Retención de nutrientes. & $\begin{array}{l}\text { Potenciales usos futuros (directos } \\
\text { e indirectos). }\end{array}$ \\
\hline Suministro doméstico e industrial. & Reducción de la contaminación. & $\begin{array}{l}\text { Valor informativo futuro } \\
\text { (biodiversidad). }\end{array}$ \\
\hline Recursos energéticos. & Estabilización de costas. & Valores de no uso. \\
\hline Transporte y navegación. & Soporte externo a ecosistemas. & Biodiversidad. \\
\hline Recreo. & Reducción del calentamiento global & Herencia cultural. \\
\hline $\begin{array}{l}\text { Pesca } \\
\text { frente a tormentas. }\end{array}$ & y estabilización microclimática. & \\
\hline
\end{tabular}

Fuente: Adaptación de varios autores realizada por Birol et al. (2006).

En términos generales, los bienes y servicios ambientales pueden ser evaluados a través de la focalización en los beneficios aportados por los mismos, o bien a través de enfoques de costes. En este último caso, se trata de una aproximación al valor de los bienes ambientales a través del cálculo de los costes de las medidas de reparación, prevención o mitigación del daño ambiental, asumiéndose que el valor del bien ambiental será al menos el valor del coste de las medidas para su protección o reparación. Esta fue la aproximación realizada en un primer momento en la aplicación del artículo 9 de la DMA relativo a la recuperación de costes en los Países Bajos y Alemania en 2004 (Brouwer (2006a); Interwies et al. (2004), respectivamente). Éste es también el enfoque que adopta en España el Ministerio de Medio Ambiente y Medio Rural y Marino (MARM) en la instrucción de la planificación hidrológica, cuando establece que los costes ambientales se valorarán como el coste de las medidas establecidas para alcanzar los objetivos ambientales, incluyendo las adoptadas tanto por las administraciones competentes como por los usuarios ${ }^{12}$.

12. Epígrafe 7.2.: Costes ambientales y del recurso, dentro del apartado de recuperación del coste de los servicios del agua. 
Sin embargo, esta aproximación de costes, que se hace en muchas ocasiones por ser relativamente más fácil y para la que existe mayor cantidad de datos disponibles, no debe confundirse con el valor del bien en sí mismo. Como señalan Brouwer y Georgiou (2007) las medidas de reparación, mitigación y prevención se derivan de la provisión del bien o servicio y no deben confundirse con la demanda, que es la que, de acuerdo con la teoría económica, determina el valor de un bien.

En el contexto concreto de la implementación de la DMA, la utilización del enfoque de costes tiene importantes limitaciones. En el caso de la recuperación de los costes del servicio del agua (artículo 9 de la DMA), la asunción de que el coste de las medidas para alcanzar los objetivos ambientales refleja la pérdida de bienestar (y por tanto los costes reales) derivada de la modificación del estado de las masas de aguas para su uso, no será siempre necesariamente correcta, ya que la recuperación del buen estado no será siempre posible. En tales casos, se estaría produciendo una infravaloración del coste ambiental (como reconocen las propias hojas informativas de la Estrategia Conjunta de Implementación de la DMA reflejadas por el ECO2) y no se estaría contabilizando el coste de oportunidad de no alcanzar el buen estado ecológico de las aguas.

Aún mayor es la limitación del enfoque de costes de las medidas de reparación y mitigación para el análisis de la posible rebaja de los objetivos ecológicos por motivos de desproporcionalidad (artículo 4): si los beneficios ambientales de la DMA (buen estado ecológico de las aguas) se equiparan al coste de las medidas necesarias para alcanzarlo, la comparación se hace simplemente incongruente, ya que se estaría incluyendo un mismo concepto a ambos lados de la ecuación ${ }^{13}$.

El enfoque alternativo, o enfoque de demanda, mide el valor que la sociedad confiere a los recursos naturales tales como el agua y a los servicios por éstos ofrecidos, mediante la noción de disponibilidad a pagar (DAP) y a aceptar (DAA) ${ }^{14}$, que tienen su origen en los derivados conceptuales del excedente del consumidor (medidas de excedente equivalente $y$

13. La instrucción de planificación hidrológica del MARM se aleja de la aproximación de costes para el caso de la desproporcionalidad, estableciendo un análisis de los beneficios derivados de alcanzar los objetivos ambientales de las masas de agua.

14. La elección entre una de estas medidas depende de la percepción de los derechos de propiedad (Freeman, 1979; Knetsch y Sinden, 1984; Hanemann, 1991; Carson, Flores y Meade, 2001). La DAP ha sido la utilizada más frecuentemente (Cummings et al., 1993; Arroz et al., 1993; Carson, 1998) porque está limitada por las restricciones presupuestarias de los encuestados. 
compensatorio) ${ }^{15}$. Con esta base, las técnicas de valoración ambiental tratan de estimar en términos monetarios las expectativas de beneficios y costes de los cambios ambientales (Romero, 1994).

Para el caso de los recursos hídricos, Loomis (2000) afirma que la valoración económica de los usos ambientales del agua permite tener una perspectiva más equilibrada de la asignación y gestión de recursos. La evaluación ambiental permite incluir los beneficios que la sociedad recibe por los usos no tradicionales del agua dentro del análisis coste-beneficio y en la estimación de los costes totales para una recuperación y uso eficiente del recurso. Debe entenderse como un mecanismo para proporcionar conocimiento adicional que permita tomar decisiones en un contexto más adecuado, permitiendo una contabilización más completa de los costes y beneficios asociados a la explotación de los recursos naturales.

La valoración económica por preferencias expresadas ${ }^{16}$, que es la única que permite de momento la estimación de valores de no uso, tiene además otra ventaja en el contexto de aplicación de la DMA que se relaciona con la vocación de participación pública de la norma europea. Por participación entendemos los foros de intercambio organizados con el propósito de facilitar la comunicación entre el gobierno, los ciudadanos, los agentes implicados, los grupos de interés en relación con una decisión o problema específico (Wittmer et al., 2006). En relación explícita con el agua, de la Declaración de Dublín emana la aceptación de que la implicación pública en todos los niveles de la toma de decisiones relativa a la gestión del agua es tanto un objetivo en sí mismo como un elemento esencial de la gestión (Green, 2003). Este mismo espíritu se encuentra en la redacción de la DMA, cuando en su artículo 14 prescribe la participación pública como un mecanismo dirigido a aumentar la transparencia y la legitimidad de la gestión del agua y ofrecer cauces de diálogo entre usuarios que faciliten la resolución de conflictos.

En el documento Guía sobre Participación Pública en relación con la DMA (Comisión Europea, 2003b) la participación pública queda definida en

15. Ambos conceptos hacen referencia a la cantidad de dinero que será necesario pagar o recibir para mantener al individuo en el mismo nivel de bienestar. En el caso del excedente compensatorio se trata de una medida previa al cambio ambiental, mientras que el excedente equivalente hace referencia a después de que ocurra el cambio ambiental (Bateman y Turner, 1993).

16. Las técnicas de valoración ambiental pueden ser por preferencias reveladas (búsqueda de bienes de mercado que reflejen en su precio las ganancias y pérdidas de bienestar asociadas al bien ambiental que se pretende valorar) y preferencias expresadas (construcción de mercados hipotéticos mediante los cuales estimar las preferencias individuales a través de encuestas) (Bateman et al., 2002). 
un sentido amplio como la facilitación de la influencia del público en el proceso de implementación ${ }^{17}$. Las encuestas (y por tanto las encuestas de valoración ambiental por preferencias expresadas) no se mencionan explícitamente en la lista de técnicas del Documento Guía, pero pueden asimilarse (o acercarse) a lo que dicho documento sí contempla: las entrevistas. Entendemos, a diferencia de Wright (2007), que las encuestas de valoración económica no deben considerarse como excluyentes de otras formas de participación pública que puedan desarrollarse como parte del proceso de implementación de la DMA.

\section{LA DEFINICIÓN DEL BUEN ESTADO ECOLÓGICO}

Para poder estimar el valor de los bienes y servicios proporcionados por el buen estado ecológico de las aguas mediante técnicas de valoración ambiental por preferencias expresada, habrá que, en un primer momento, tener claro qué entendemos por buen estado ecológico y, en un segundo momento, cómo definirlo en términos entendibles por el público que responderá a las encuestas.

El artículo 2(21) de la DMA define el estado ecológico como una expresión de la calidad de las estructuras y funcionamiento de los ecosistemas asociados con las aguas superficiales pero sólo proporciona una definición a rasgos generales del estado ecológico en de acuerdo con parámetros biológicos, hidromorfológicos y físico-químicos. Concretamente, para el caso de las aguas superficiales, define el "buen estado" como aquél estado de las masas de agua en el que los indicadores de calidad biológicos muestran valores bajos de distorsión causada por la actividad humana, pero sólo se desvían ligeramente de los valores normalmente en condiciones inalteradas ${ }^{18}$. La Directiva define a su vez el "muy buen estado" (condiciones inalteradas), "estado aceptable" (desviaciones moderadas) y los "estados deficiente y malo" (condiciones por debajo del estado aceptable).

En noviembre de 2002 un grupo de trabajo informal dedicado a la definición del estado ecológico de las aguas superficiales, el ECOSTAT WG 2.A, fue creado en el contexto de la Estrategia Conjunta de Implementación de la DMA con la responsabilidad del desarrollo de directrices de apoyo para

17. Siguiendo la escala de participación propuesta por Arnstein (1969), el documento guía distingue entre: i) provisión de información, ii) consulta, y iii) participación activa.

18. Directiva Marco del Agua, anexo V: definiciones normativas de las clasificaciones del estado ecológico. 
el proceso de clasificación ecológica, es decir, establecimiento de los parámetros y umbrales que definen el estado ecológico y los distintos niveles. Como consecuencia de los trabajos del ECOSTAT se creó el documento del Enfoque General para la Clasificación del Estado y Potencial Ecológico (Comisión Europea 2003c), en el que se resumen las reglas y foco generales para esta clasificación. Cuatro años después de estas reflexiones, en el contexto del Ejercicio Europeo de Intercalibración, se admite que, a pesar del ingente trabajo que actualmente se está realizando, no existe todavía una garantía final relativa a la ambición de establecer niveles de estado ecológico comparables entre los países.

Es decir, existe todavía una falta de consenso a cerca de los parámetros físico-químicos y biológicos que definen, en el contexto de la DMA, el buen estado ecológico y los distintos niveles. Desde el ámbito económico nos encontramos pues ante un doble problema: i) la imposibilidad de "esperar" a dicho acuerdo sobre la definición de buen estado ecológico (recordamos el imperativo de que los Planes de cuenca deben ser publicados para finales de 2009), y ii) la necesidad de que, en cualquier caso, los escenarios de valoración económica (por preferencias expresadas) deben no sólo responder a las demandas informativas requeridas por la DMA para su inserción como insumos de la política pública (es decir, debe ser entendibles para el planificador), sino que también debe ser relevantes y comprensibles para el público que deberá valorar los bienes y servicios.

En esta línea, Hanley et al. (2006a) han probado el siguiente enfoque: la definición del estado ecológico mediante la utilización de parámetros tipo físico y ecológico (vida acuática, presencia de basuras y calidad de las riberas) para valorar los beneficios ambientales derivados de la mejora del estado de las aguas. Plantearon la valoración mediante un experimento de elección de la calidad ecológica de los ríos en base a dichos parámetros. Sin embargo, hay que señalar que estos atributos resultaron no significativos en la valoración, lo que los autores atribuyen posiblemente a que dichos atributos no resultaron comprensibles por el público.

Un enfoque alternativo en este sentido sería el que se propone en Martín-Ortega et al. (en prensa) en el que calidad ambiental del agua y sus distintos niveles se definen en términos de los posibles usos del agua a modo de "escala" de usos (calidad muy buena, buena, moderada y mala). Esta definición se basa en la escala de niveles de calidad propuesta por Carson y Mitchell (1993), quienes prepararon para la Agencia de Medio Ambiente estadounidense un estudio de valoración del agua en términos de su susceptibilidad de uso, es decir, la valoración de la calidad de un agua que 
garantizara las posibilidades de baño (swimminable), pesca (fishable) y navegación (boatable). Estos usos están, como en el estudio de Carson y Mitchell, así como en estudios posteriores como el de Herberling (2000), organizados de forma jerárquica, de modo que cada nivel superior dentro de la escala incluye los usos incluidos en los niveles inferiores.

El trabajo de Carson y Mitchell no está exento de críticas. Este enfoque basado en la escala de categorías de calidad vinculadas al uso del agua asume una determinada ordenación jerárquica de los usos. Existen trabajos que abogan por que esta simplificación jerárquica no se corresponde siempre con las certidumbres científicas sobre la calidad hídrica e insisten en la necesaria complejidad multidimensional de la valoración del agua. Tales son las conclusiones de Wesley et al. (2000) quienes encuentran en su estudio basado en estadísticas de la EPA estadounidense sobre lagos y ríos, que en el $85 \%$ de los casos estudiados, el agua es susceptible de ser bebida pero sólo el $79 \%$ es recomendable para baño, violando la jerarquía de Carson y Mitchell. Sin embargo, entendemos que esta aproximación ha dado mejores resultados y que sirve a los propósitos de la DMA.

Finalmente, consideramos además, que la magnitud del cambio en la provisión del bien ambiental determinará el nivel de utilidad que de ella se deriva. Es por ello que en las estimaciones que se hagan será necesario desarrollar mecanismos de valoración que sean sensibles a diferentes incrementos de la calidad y capaces de detectar estos impactos diferenciales en el bienestar, lo que se conoce como sensibilidad al alcance (Carson, 1997).

\section{IMPORTANCIA DE LA VARIABLE TERRITORIAL}

El agua se genera y fluye superficial o subterráneamente por un determinado territorio con el que interacciona. Moral Ituarte (1994) describe dos tipos de relaciones agua-territorio normalmente conectadas y superpuestas: las que se refieren al aprovechamiento del recurso y las que afectan a su generación, conservación y circulación, de lo que se desprende que el recurso hídrico presenta una doble entidad: el agua propiamente dicha y el espacio que permanente o discontinuamente ocupa. Como consecuencia de lo anterior, los conflictos generados en torno al agua tienen una dimensión territorial ineludible: no son sólo intersectoriales sino también territoriales, tanto en lo referido a la cantidad como a las repercusiones sobre la calidad del agua y los cauces (contaminación en la parte alta de las cuencas frente a las consecuencias en las partes bajas, por ejemplo). 
La DMA ofrece una nueva oportunidad para responder a la necesidad de que la planificación hidrológica se integre en un modelo de desarrollo territorial más amplio ${ }^{19}$. La DMA establece la demarcación hidrográfica como la base esencial del modelo de la política hídrica, imponiendo un enfoque geográfico-físico (y por tanto territorial) para la definición de los objetivos e instrumentos de gestión, abandonando el enfoque de tipo administrativo de las fases previas de su redacción (González-Antón y Arias, 2001). De hecho, la primera tarea que la Directiva impone a los Estados Miembros es la identificación y designación de las Demarcaciones Hidrográficas (artículo 3), que define como la zona marina y terrestre compuesta por una o varias cuencas hidrográficas vecinas, más las aguas subterráneas y costeras asociadas y establece una serie de elementos para la administración y planificación de las mismas, incluida la creación de una Agencia o autoridad competente 20 . La DMA requiere que las demarcaciones hidrográficas sean tratadas como un todo en el seno de los planes de gestión integrada (Hanley et al., 2006a).

Entre los contenidos hidrológicos que la ordenación territorial debería incluir a juicio de Moral Ituarte (2001) se encuentran la definición de objetivos de calidad en relación con los diferentes usos de cada zona, la definición de zonas sensibles y los aspectos territoriales de la evaluación de los recursos. En relación con este último elemento, el autor hace referencia a las posibilidades de regulación superficial y subterránea, pero entendemos que los argumentos son también válidos para la evaluación de otros aspectos del agua, como el de los beneficios ambientales. El autor estima que el debate sobre las obras de regulación es cada vez más un debate sobre la relación entre territorios. La idea que subyace a la propuesta que aquí planteamos es que esta relación entre territorios deberá ser tenida en cuenta no sólo en las obras de regulación sino en la consecución de los objetivos ecológicos de la Directiva y por tanto, la dimensión territorial debe ser incluida en el análisis de los beneficios y costes ambientales, ya que la representación del capital natural en el espacio contribuye a proporcionar

19. En España, los debates surgidos sobre el Plan Hidrológico Nacional (2001) y sobre el reparto de competencias de la gestión de cuencas en la reforma estatutaria, así como la evidencia de un urbanismo agresivo y en ocasiones fraudulento que ignora criterios hídricos, ponen de manifiesto que la planificación hidrológica está pendiente aún de ser integrada al servicio de la política territorial.

20. Para el caso español, esta primera imposición comunitaria ya estaba cubierta por la existencia de las Confederaciones Hidrográficas desde 1926. Para ver las novedades incluidas por la Directiva en el tema de autoridades de gestión se puede consultar a Piñero Campos (2004). 
indicadores más ajustados de los efectos en el bienestar de cambios ambientales (Brody y Highfield, 2004).

Por todo ello, entendemos que no sólo la magnitud de la mejora de la calidad ambiental influye en la utilidad derivada del mismo y por tanto en los beneficios ambientales: también es importante el lugar donde dicha mejora ocurre y la escala en la que se produce en relación con el individuo. De manera específica al asunto que nos ocupa, dos son las razones por las que consideramos que la investigación de los beneficios ambientales debería incluir la dimensión territorial: i) la desigual distribución de los beneficiarios de las mejoras inducidas por la implantación de la norma, y ii) las necesidades de transferencia de resultados.

Por una parte y como señalan Bateman et al. (2006a), los efectos de la implementación de la DMA no serán geográficamente homogéneos y los beneficios derivados de la mejora de la calidad de las aguas variarán en el espacio no sólo según la distribución y la respuesta física de las masas de agua, sino también en el sentido de la distribución de los beneficiarios. Los análisis económicos que se derivarán de la implementación de la DMA requerirán la agregación de los beneficios individuales para que puedan ser comparados con los costes totales de las medidas o políticas de gestión. El modo en que se realice la agregación será absolutamente crucial y deberá hacerse sobre la base de la llamada jurisdicción económica, es decir, aquéIla que incluye a la población beneficiara de la medida, frente a la jurisdicción política o administrativa (Bateman et al., 2006b). La manera de evitar los sesgos derivados del proceso de agregación pasa necesariamente por la identificación de funciones de valoración espacialmente sensibles.

En el ámbito de la economía ambiental y de los recursos naturales el espacio, en forma de distancia, ha jugado un papel importante en el análisis de la demanda recreativa (el método del coste del viaje está basado de forma esencial en términos espaciales) y en modelos de precios hedónicos de la tierra (Bateman et al., 2002) pueden ser consultados en relación a los elementos clave y últimos avances en este sentido. Sin embargo, ya a mediados de los noventa, algunos autores como Eade y Moran (1996) y Bockstael (1996), subrayaron que se había prestado relativamente poca atención a la dimensión espacial en el análisis de la sostenibilidad y las relaciones entre la distribución del capital natural y el bienestar. Una década después, la literatura científica sigue poniendo de manifiesto que el análisis de la escala espacial en la que los servicios de los ecosistemas son proporcionados y su implicación para los distintos agentes, sigue siendo escaso (Hein et al., 2006; Bateman et al., 2002). Schaafsma y Brouwer (2006) 
encuentran, en su revisión de guías de valoración ambiental aplicadas a los recursos hídricos, que los aspectos espaciales y territoriales han sido hasta el momento considerados de manera sólo limitada. Mientras que muchas de estas guías para la valoración ambiental apuntan a la importancia de la especificidad del emplazamiento, apenas ninguna de ellas discute la posible dependencia espacial de los valores relativos al agua. Johnston et al. (2002) encuentran que los estudios de preferencia expresada raramente incorporan atributos espaciales que son, sin embargo, relevantes para la estimación del bienestar.

Por otra parte, la inclusión de la dimensión espacial en la valoración de los beneficios ambientales derivados de la aplicación de la DMA tiene un aliciente adicional en la exploración de las posibilidades de transferencia de resultados. La transferencia de resultados es una técnica en las que los resultados de estudios ambientales previos se aplican a nuevos contextos. Se define como la transposición de valores monetarios ambientales estimados en un lugar (lugar de estudio) a otro lugar (lugar de aplicación). Hanley et al. (2006b) constatan, tras analizar los estudios que desde hace ya una década prueban la transferencia de resultados y evalúan su precisión, que el consenso a cerca de la validez de estas técnicas en el mundo académico no está todavía alcanzado. Sin embargo, consideramos que su aplicación se postula como particularmente necesaria en el contexto de la aplicación de la DMA, ya que parece difícilmente imaginable una plena implementación de la norma, al nivel y en los plazos que la misma requiere, sin la utilización de métodos de valoración extensivos, como la transferencia de valores. Consideramos que la inclusión de la dimensión territorial como atributo de la valoración de los beneficios de aplicación de la DMA puede suponer un paso adelante en la mejora de estas técnicas.

\section{CONCLUSIONES}

La Directiva Marco del Agua establece la mejora del estado de las masas europeas desde la situación actual hasta el buen estado ecológico y requiere explícitamente la incorporación de los beneficios y costes ambientales en la gestión hídrica. Sin embargo, la definición práctica de estos conceptos no ha sido todavía suficientemente clarificada ni desde el ámbito institucional ni desde el ámbito científico y los avances de los Estados Miembros para su estimación son todavía reducidos. La escasez de 
trabajos aplicados adquiere tintes de gravedad si tenemos en cuenta que en el año 2009 deben estar aprobados los Planes de cuenca, y que en ellos debería argumentarse (artículo 4) la existencia de costes desproporcionados.

Los modelos económicos que se apliquen para el análisis coste-beneficio de la aplicación de la Directiva deberán ser capaces de estimar el valor derivado de tal cambio en el estado del agua, incluido los valores uso directo, uso indirecto y no uso, para los que no existe un mercado, con el fin de corregir una situación de asignación ineficiente del recurso. Estas evaluaciones económicas deberán superar el enfoque reduccionista de la aproximación de los costes de reparación, mitigación y prevención del daño ambiental, que resultan incongruentes en el caso de la DMA, particularmente para la posible derogación de los objetivos ecológicos. En este sentido, la valoración ambiental tiene un rol importante a jugar, como mecanismo para identificar las preferencias del público en materia de calidad del agua y como mecanismo coherente con la vocación de participación pública de la Directiva. Entendemos que la valoración monetaria contribuye a una asignación más eficiente del recurso (incluyendo los valores del recurso no estrictamente reflejados en el mercado) mediante la incorporación al proceso de toma de decisiones de una información más completa sobre los beneficios y costes sociales que el agua ofrece. No debe entenderse en esta propuesta necesariamente un rechazo a enfoques más ecológicos o participativos, sino una intención de buscar soluciones rigurosas adaptadas al tempo de la Directiva.

Los escenarios de valoración que se empleen deberán, por una parte, ser sensibles a las distintas magnitudes de cambio en la mejora del estado del agua (hay que tener en cuenta que se parte de situaciones iniciales muy diferentes entre las distintas cuencas e incluso entre las distintas masas de agua que componen dichas cuencas), es decir, ser capaces de identificar beneficios crecientes ante mayores niveles de provisión del bien ambiental. Pero también, el cambio de estado del agua no puede ser desvinculado del territorio en el que se produce (y así debe reflejarse en el ejercicio de valoración). La inclusión de la variable territorial (apoyada por ejemplo en la utilización de sistemas de información geográfica en su aplicación territorial) contribuirá a la obtención de estimaciones más ajustadas del bienestar.

Recibido 17.12.07

Aceptado 15.12.08 


\section{BIBLIOGRAFÍA}

Álvarez-Farizo, B.; Hanley, N.; Barberán, R. y Lázaro, A. (2006): "Choice Modelling at the 'Market Stall': Individual versus Collective Interest in Environmental Valuation", Ecological Economics.

Álvarez-Farizo, B. y Hanley, N. (2006): "Improving the Process of Valuing Non-Market Benefits: Combining Citizens Juries with Choice Modelling", Land Economics, vol. 82, n 3, pp. 465-478.

Andreu, J.; Pulido, M.; Collazos, G. y Pérez, M. A. (2006): Metodologías y Herramientas para el Análisis Económico de Sistemas y Recursos Hídricos. Aplicación a la DMA, Fundación Nueva Cultura del Agua (Editor), Congreso Ibérico sobre gestión y planificación del agua.

Arnstein, S. R. (1969): "A Ladder of Citizen Participation", Journal of the American Institute of Planners, 35, pp. 216-224.

Azqueta, D. y Delacámara, G. (2006): "Ethics, Economics and Environmental Management", Ecological Economics, vol. 56, n 4 , pp. 524-533.

Baker, B.; Metcalfe, P.; Butler, S. et al. (2007): Report on the Benefits of Water Framework Directive Programmes of Measure in England and Wales, DEFRA, Reino Unido.

Bateman, I. J.; Carson, B. y Day, B. H. (2002): Economic Valuation with Stated Preference Techniques: Summary Guide, Edgar Elgar, Massachussets.

Bateman, I. J.; Brouwer, R.; Davies, H.; Day, B. H.; Deflandre, A.; Falco, S. D.; Georgiou, S.; Hadley, D.; Hutchins, M.; Jones, A. P.; Kay, D.; Leeks, G.; Lewis, M.; Lovett, A. A.; Neal, C.; Posen, P.; Rigby, D. y Kerry Turner, R. (2006a): "Analysing the Agricultural Costs and Non-market Benefits of Implementing the Water Framework Directive", Journal of Agricultural Economics, 57, pp. 221-237.

Bateman, I. J.; Day, B. H.; Georgiou, S. y Lake, I. (2006b): "The Aggregation of Environmental Benefit Values: Welfare Measures, Distance Decay and Total WTP", Ecological Economics, 60: 450-460.

Birol, E.; Karousakis, K. y Koundouri, P. (2006): “Using Economic Valuation Techniques to Inform Water Resources Management: A Survey and Critical Appraisal of Available Techniques and an Application", Science of the Total Environment, 365, pp. 105-122.

Bockstael, N. (1996): "Modelling Economics and Ecology: the Importance of a Spatial Perspective", American Journal of Agricultural Economics, vol. 78, December, pp. 1168-1180.

Brody, S. D. y Highfield, L. A. (2004): “Does Location Matter? Measuring Environmental Perceptions of Creeks in Two San Antonio Watersheds", Environment and Behaviour, 36, pp. 229-250.

Brouwer R. (2006a): Assessment of Environmental and Resource Cost in the Water Framework Directive, International Workshop on Hydro-economic Modelling and Tools for Implementation of the EU Water Framework Directive, Valencia.

Brouwer R. (2006b): "Valuing Water Quality Changes in the Netherlands using Stated Preference Methods", en DW Pearce (ed.), Valuing the environment in Developed Countries, Edward Elgar Publishing, Cheltenham, Reino Unido.

Brouwer, R. y Georgiou, S. (2007): Economic Valuation of Environmental and Resources Costs and Benefits of Water Uses and Services in Water Framework Directive: Technical Guidelines for Practitioners, EU $6^{\text {th }}$ FP AquaMoney Project: Development and Testing of Practical Guidelines for the Assessment of Environmental and Resource Costs and Benefits in the WFD: www.aquamoney.org

Brouwer, R. (2008): "The Role of Stated Preference Methods in the Water Framework Directive to Assess Disproportionate Costs", Journal of Environmental Planning and Management, 51(5), pp. 597-614.

Carson, R. T. y Mitchell, R. (1993): "The value of clean water: the public's willingness to pay for boatable, fishable and swimmable quality water", Water Resources Research, 29, p. 2445. 
Carson, R. T. (1997): Contingent Valuation Surveys and Tests of Insensitivity to Scop, en Kopp, R. J.; Pommerhene, W. y Schwartz, N. (eds.), Determining the Value of Non-marketed Goods: Economic, Psychological and Policy Relevant Aspects of Contingent Valuation Methods, Kluwer, Boston, pp. 127-163.

Carson, R. T.; Flores, N. E. y Meade, N. F. (2001): "Contingent Valuation: Controversies and Evidence", Environmental and Resource Economics, 19, p. 173-210.

Comisión Europea (2003a): Economics and the Environment: The implementation Challenge of the Water Framework Directive, A Guidance Document, WATECO.

Comisión Europea (2003b): Guidance Document $n^{\circ}$ 8, Public Participation in Relation to the Water Framework Directive. Common Implementation Strategy for the Water Framework Directive, Bruselas.

Comisión Europea (2003c): Overall approach for the classification of Ecological Status and Ecological Potential, Office for Official Publications of the European Commission, Luxemburg.

Costanza, R. (1989): "What is Ecological Economics?", Ecological Economics, 1, p. 1.

Costanza R.; D'Arge, R.; Groot, R.; Farber, S.; Grasso, M.; Hannon, B.; Limburg, K.; Naeem, S.; O'Neil, R.; Paruelo, J.; Raskin, R.; Sutton, R. y Van den Belt, M. (1997): "The Value of the World's Ecosystem Services and Natural Capital", Nature, 387, pp. 253-260.

Cummings R. G.; Brookshire, D. S. y Schulze, W. D. (1993): Valuing environmental goods: an assessment of the contingent valuation method, Rowman and Allanheld, Totowa.

Eade, J. D. O. y Moran, D. (1996): "Spatial Economic Valuation: Benefits Transfer using Geographical Information Systems", Journal of Environmental Management, vol. 48, n² 2, pp. 97-110.

ENCOBALT (2007): Valuing environmental costs and benefits for supporting the implementation of the WFD in the Baltic Member States.

Ferrer, G. y La Roca, F. (2006): El papel de la economía en el desarrollo e implementación de la Directiva Marco del Agua. Ambigüedad conceptual y problemas prácticos, Universidad de Valencia, Mimeo.

Freeman, A. M. I. (1979): The benefits of environmental improvement: Theory and practice, The John Hopkins University Press, Publisher.

Gómez, C. M. (2006): Los costes y beneficios ambientales en el proceso de decisión de la DMA, Hydronomic Convention, Barcelona.

González-Antón, C. y Arias, C. (2001): "The Incorporation of Integrated Management in European Water Policy", Integrated Water Resources Management, IAS Press, Oxfordshire, pp. 69-74.

Görlach, B. e Interwies, E. (2006). "Assessing Environmental and Resource cost in the Water Framework Directive: the Case of Germany", Ecologic, Institut für Internationale und Europäische Umweltpolitik, Berlín.

Green, C. (2003): Water Economics, Principles and Practice, Wiley, West Sussex.

Hanemann, W. M. (1991): "Willingness to Pay and Willingness to Accept: How Much Can They Differ?", The American Economic Review, 81, pp. 635-647.

Hanley, N.; Wright, R. y Álvarez-Farizo, B. (2006a): "Estimating the economic value of improvements in river ecology using choice experiments: an application to the water framework directive", Journal of Environmental Management, 78, pp. 183-193.

Hanley, N.; Colombo, S.; Tinch, D.; Black, A. y Aftab, A. (2006b): "Estimating the Benefits of Water Quality Improvements under the Water Framework Directive: are Benefits Transferable?", Eur Rev Agric Econ, 33, pp. 391-413.

Heberling, M.; Fisher, A. y Shortle, J. (2000): How the number of choice steps affects responses in stated choice surveys, US EPA, Cincinnati.

Hein, L.; van Koppen, K. R.; De Groot, R. S. y van lerland, E. C. (2006): "Spatial Scales, Stakeholders and the Valuation of Ecosystem Services", Ecological Economics, vol. 57, n' 2, pp. 209-228. 
Interwies, E.; Kraemer, A.; Kranz, N.; Görlach, B. y Dworak, T. (2004): Basic Principles for Selecting the Most Cost-Effective Combinations of Measures for Inclusion in the Programme of Measures as Described in Article 11 of the Water Framework Directive, Handbook, German Federal Environmental Agency, Berlín.

Johnston, R. J.; Swallow, S. K. y Bauer, D. M. (2002): "Spatial Factors and Stated Preference Values for Public Goods: Considerations for Rural Land Use", Land Economics, vol. 78, pp. 481-500.

Knetsch, J. L. y Sinden, J. A. (1984): "Willingness to Pay and Compensation Demanded: Experimental Evidence of an Unexpected Disparity in Measures of Value", The Quarterly Journal of Economics, 99, pp. 507-521.

Loomis, J. B. (2000): "Environmental Valuation Techniques in Water Resources Decision Making", Journal of Water Resources Planning and Management, pp. 339-344.

Martínez Alier, J. y Roca Jusmet, J. (2000): Economía Ecológica y Política Ambiental, Fondo de Cultura Económica, México.

Ministerio de Medio Ambiente y Gobierno de Navarra (2002): Análisis Económico del Plan de Cuenca del Cidacos, Aplicación de la Guía de Análisis Económico de la Directiva Marco del Agua.

Moral Ituarte, L. (1994): "Elementos para una teoría de los conflictos territoriales sobre el agua", Boletín de la A.G.E., 18, pp. 17-27.

Moral Ituarte, L. (2001): "El tratamiento del agua en la ordenación del territorio. consideraciones sobre su concreción en Andalucía", Scripta Veterea, 81.

Naciones Unidas (2003): Informe sobre el desarrollo de los recursos hídricos en el mundo: agua para todos, agua para la vida.

Pearce, D. W. y Turner, R. K. (1995): Economía de los recursos naturales y el medio ambiente, Colegio de Economistas de Madrid, Madrid.

Piñero Campos, J. M. (2004): “La Transposición de la Directiva Marco de las Aguas al Derecho Español", Jornadas sobre La Aplicación de la Directiva Marco del Agua, Colegio de Ingenieros de Caminos Canales y Puertos, Madrid.

Postle, M.; Foottit, A.; Fenn, T. y Salado, R. (2004): CEA y Developing a Methodology for Assessing Disproportionate Costs, Department for Environment, food y Rural Affairs (DEFRA), Reino Unido.

Romero, C. (1994): Economía de los Recursos Ambientales y Naturales, Alianza Economía, Madrid.

Schaafsma, M. y Brouwer, R. (2006): "Overview of Existing Guidelines and Manuals for the Economic Valuation of Environmental Costs and Benefits", EU $6^{\text {th }}$ FP AquaMoney Project: Development and Testing of Practical Guidelines for the Assessment of Environmental and Resource Costs and Benefits in the WFD: www.aquamoney.org

Strosser, P. (2006): Assessing environmental costs in the context of the implementation of the European Union Framework Directive, Barcelona.

UNESCO (2002): 21st Century Talks, París.

Unión Europea (2000): Directiva 2000/60/CE por la que se Establece el Marco Comunitario de Actuación en Política de Agua.

Wesley A. M.; Joel, H.; Viscusi, W. K. y Jason, B. (2000): "An Iterative Choice Approach to Valuing Clean Lakes, Rivers, y Streams", Journal of Risk and Uncertainty, vol. 21, pp. 7-43.

Wittmer, H.; Rauschamyer, F. y Klauer, B. (2006): "How to Select Instruments for the Resolution of Environmental Conflicts?", Land Use Policy, 23, pp. 1-9.

World Wide Fund for Nature and European Bureau (2006): Survey of the economic elements of the Article 5 report of the EU Water Framework Directive, Bruselas.

Wright, S. A. L. (2007): Decision-making in the European Water Framework Directive: the potential consequences of the neoclassical approach, Informe 5.958.473, Facultad de Ciencias de la Vida, Universidad de Copenhague: www.forskiningsdatabasen.dk 


\title{
Resumen
}

Dentro del análisis económico que la Directiva Marco del Agua (DMA) prescribe para alcanzar una gestión sostenible de los recursos hídricos, destaca la necesidad de evaluar los beneficios y costes ambientales. Dada la falta de una definición explícita de estos conceptos en el texto de la Directiva y, al mismo tiempo, la necesidad acuciante de evaluar la desproporcionalidad de los costes de la implementación de la DMA, este artículo pretende contribuir a la reflexión sobre los conceptos de beneficio y coste ambiental en este contexto. Se discuten la diferentes interpretaciones y valoraciones que se han hecho hasta el momento en Europa y se profundiza en aspectos claves para su estimación.

Palabras clave: Directiva Marco del Agua; costes y beneficios ambientales; costes desproporcionados.

\begin{abstract}
Environmental costs and benefits play a major role in the economic analysis prescribed by the Water Framework Directive for the achievement of a sustainable management of water resources. The definition of these concepts is not explicit in the text of the Directive, while there is an urgent need to apply then for analysing whether the implementation of the WFD is disproportionately costly. This paper tries to contribute to the reflexion on the concepts of environmental costs and benefits in the context of the WFD. We discuss the different interpretations and valuations that have been made up to date in Europe and some of the key issues for their estimation.
\end{abstract}

Key words: Water Framework Directive; environmental costs and benefits; disproportionate costs. 